\title{
EFECTOS DE UN PROGRAMA PARA EL MEJORAMIENTO DE LA AUTOESTIMA EN NIÑOS DE 8 A 11 AÑOS CON PROBLEMAS ESPECÍFICOS DE APRENDIZAJE
}

\author{
Mg. Juan Pequeña Constantino*, Mg. Luis Miguel Escurra Mayaute**
}

\section{RESUMEN}

Se elaboró un programa de autoestima y se evaluó el mejoramiento de la autoestima en niños de 8 a 11 años con problemas específicos de aprendizaje. Se tuvieron 30 participantes (15 grupo experimental y 15 grupo control).

Se utilizó un diseño experimental con grupo control pre test y post test. En el análisis comparativo se encontró que existen diferencias significativas en el área de sí mismo $(\mathrm{t}=$ $-4.06, \mathrm{p}<.01)$ hogar $(\mathrm{t}=-2.26, \mathrm{p}<.05)$ escuela $(\mathrm{t}=-3.45, \mathrm{p}<.01)$ pares $(\mathrm{t}=-$ $3.06, \mathrm{p}<.01)$ y en el total de autoestima $(\mathrm{t}=-6.23, \mathrm{p}<.001)$ notándose en todos los casos que el post test es mayor que el pre test, lo que corrobora la efectividad del programa de mejoramiento de la autoestima.

Palabras clave: Autoestima, programa, niños, problemas específicos de aprendizaje.

\begin{abstract}
It was a program of self-esteem for childrens of 8 to 11 year old with specific learning disorders. They were 30 participants ( 15 were in the experimental group and 15 were in the control group).

It was used an experimental design pre test and post test with control group. In the comparative analysis it was found that there were significative differences in the area itself $(\mathrm{t}=-4.06, \mathrm{p}<.01)$ home $(\mathrm{t}=-2.26, \mathrm{p}<.05)$ school $(\mathrm{t}=-3.45, \mathrm{p}<.01)$ mats $(\mathrm{t}=$ $-3.06, \mathrm{p}<.01)$ and in total self esteem $(\mathrm{t}=-6.23, \mathrm{p}<.001)$, in all cases the post test was greater than pre test, this results corroborate the efectiveness of the program
\end{abstract}

Keywords: Self-esteem, Program, children, specific of learning disorders.

* Docente Asociado, Facultad de Psicología de la UNMSM. E-mail: jpequeñac@unmsm.edu.pe

** Docente Principal, Facultad de Psicología de la UNMSM. E-mail: mescurra@peru.com 


\section{INTRODUCCION}

La autoestima no es un concepto abstracto, alejado del quehacer cotidiano de las personas. Por el contrario, casi todos los actos de nuestras vidas están marcados por el nivel que hemos logrado desarrollar. Esta se manifiesta permanentemente a través de nuestras conductas, apreciaciones y hasta en nuestra postura corporal y actitudinal.

Branden (1994) la define como la experiencia fundamental de que podemos llevar una vida significativa y cumplir sus exigencias. En otras palabras, la autoestima es la confianza en nuestra capacidad de pensar, en nuestra capacidad de enfrentarnos a los desafíos básicos de la vida, por un lado; y la confianza de nuestro derecho a triunfar y a ser felices, por el otro.

Otra definición que clarifica el significado de autoestima, es aquella que brindó la Comisión del Estado de California en 1984, con motivo de institucionalizar la enseñanza de la misma en las escuelas primarias y secundarias. Esta comisión definió la autoestima como la apreciación de la propia valía e importancia y la asunción por el individuo de su responsabilidad hacia sí mismo y hacia sus relaciones intra e interpersonales.

Es importante hacer hincapié en el hecho de que la autoestima a pesar de que reúne un conjunto de características y actitudes de carácter interior de las personas, se manifiesta y se desarrolla habitualmente dentro del contexto de su vida social, como la familia, la escuela, el trabajo, etc. De allí que la autoestima, no puede ser considerada simplemente como un sentimiento de autosatisfacción, debido a la gran relevancia que también tiene en las relaciones con los demás y con el entorno. Analizando con mayor profundidad la definición que se ha considerado surge la necesidad de conocer primero que significa apreciar la propia valía e importancia y luego que implica el ser responsable hacia sí mismo y hacia los demás.

Reconocer y apreciar la propia valía e importancia significa ser conscientes, no sólo de la propia forma de actuar en un momento dado, sino también de los propios potenciales y posibilidades. Esto lleva a entender nuestra actuación como el producto y al mismo tiempo la causa de un aprendizaje continuo y de una toma de conciencia de que estamos aprendiendo cada día más.

El ser responsable hacia sí mismo significa aceptar que cada uno asume concientemente las propias acciones y, por lo tanto, también las consecuencias de las mismas. En cuanto responsables de algo, si este algo no nos gusta podemos también responsabilizarnos de su modificación. Asumir nuestra responsabilidad con nosotros mismos implica, pues, confiar en nuestra propia capacidad para evaluar y hasta para crear nuestra realidad.

Del mismo modo, la responsabilidad de nuestras relaciones con los demás presupone aceptar, reconocer y apreciar su valía e importancia, además de la nuestra. Cuando somos conscientes de nuestra propia unicidad como personas, aprendemos a respetar el hecho de que los demás también son únicos. Como consecuencia nos abrimos a la aceptación de cada uno por lo que es y ponemos las bases para unas relaciones dirigidas a sentirnos cómodos a aprender y a facilitar que los demás también lo hagan, aceptando que son, lo mismo que nosotros, aunque en un grado o nivel distinto, personas en proceso de crecimiento, aprendizaje y maduración.

Resumiendo lo anteriormente expuesto se puede concluir diciendo, que la autoestima es la valoración y el conocimiento que cada persona tiene de sí misma, así como el sentido de 
responsabilidad personal por el desarrollo de su relación consigo mismo y con los demás. Esta se expresa en una actitud de aprobación o desaprobación, que revela el grado en que una persona se siente y se sabe capaz, exitoso y digno, actuando como tal.

La autoestima supone la adopción de una actitud hacia uno mismo y presenta tres componentes básicos:

a. Componente cognitivo: las ideas, las opiniones, las creencias, la percepción y el procesamiento de la información.

b. Componente afectivo: admiración de la propia valía personal.

c. Componente conductual: intención de actuar, llevar a la práctica un comportamiento consecuente.

\section{Importancia de la Autoestima}

El nivel de la autoestima tiene profundas consecuencias en cada aspecto de nuestra existencia: en el ámbito personal, familiar, escolar y social.

Existen correlaciones positivas entre la autoestima favorable y otros rasgos que están relacionados directamente con la capacidad para conseguir lo que nos proponemos: metas ideales, objetivos, etc.

Una autoestima saludable, como sostiene Branden (1994), se relaciona con la racionalidad, el realismo, con la creatividad, la independencia, la flexibilidad y la capacidad para aceptar los cambios, con el deseo de admitir los errores y la disposición a cooperar. Mientras que una autoestima baja se correlaciona con la irracionalidad y la ceguera ante la realidad, con la rigidez, el miedo a lo nuevo, con la conformidad, con la sumisión o el comportamiento reprimido y la hostilidad a los demás: todas estas características tienen implicancias para la realización personal y la adaptación social.

Cuanto más sólida es la autoestima, mejor preparado está el sujeto para hacer frente a los problemas que se presentan a nivel personal, familiar o laboral. Cuanto mayor sea el nivel de autoestima, la forma de comunicación será más abierta, honrada y apropiada porque creemos que nuestros pensamientos tienen valor al igual que los ajenos. Del mismo modo, nos inclinaremos a tratar a los demás con respeto, buena voluntad y justicia, y que no tendremos a considerarlos amenaza, pues el respeto a uno mismo es el fundamento del respeto a los demás.

Alcántara (1988) señala también implicancias significativas de la autoestima como núcleo de la personalidad. En este sentido la autoestima:

a. Condiciona el aprendizaje, pues la baja autoestima genera impotencia y frustración en los estudios. Las bajas calificaciones pueden reforzar sentimientos de incapacidad propia frente a su rendimiento. Por el contrario, cuando se promueven actividades que favorecen una alta autoestima, el rendimiento escolar mejora notoriamente y se abordan los nuevos aprendizajes con confianza y entusiasmo.

b. Ayuda a superar las dificultades personales, así los fracasos y conflictos no serán experiencias paralizantes, pues primarán sobre estos la estima personal y la seguridad en las propias capacidades. 
c. Fundamenta la responsabilidad, pues quienes se valoran positivamente se comprometen con mayor facilidad y desarrollan un sentido amplio de responsabilidad en las actividades que realizan.

d. Apoya la creatividad, pues la fluidez, originalidad de ideas e incentivos se consiguen con una adecuada autovaloración.

e. Determina la autonomía personal, ya que la consolidación de una autoimagen positiva, permite la formación de personas autónomas, independientes y seguras.

f. Permite relaciones sociales saludables, abiertas y asertivas.

g. Garantiza la proyección futura de la persona, pues en la medida que nos valoramos crecen las expectativas por nuestro desempeño sea escolar, laboral, social, etc. Aspirando a metas superiores.

\section{Importancia de la autoestima en la educación}

Los niños llegan al colegio portando dentro de sí las consecuencias de las relaciones que mantienen con los adultos que más significado tienen para ellos en la vida. La relación más importante es, sin duda alguna, la que sostienen con sus padres. A estos niños también les afectarán las experiencias que tengan con sus abuelos (especialmente si viven bajo el mismo techo), tías, tíos y cuidadores. Estas relaciones constituyen el espejo a través del cual el niño o la niña desarrolla su autoestima. Cuando ambos alcanzan la edad escolar ya tienen formada una imagen de sí mismos, imagen que puede alterarse por sucesivas experiencias con profesores y compañeros de clase.

Ahora se sabe que, con frecuencia, los niños que tienen dificultades de aprendizaje en el colegio tiene también problemas de autoestima y que es necesario que antes experimenten una elevación de la misma si se quiere que estos niños aumenten su rendimiento escolar. Los profesores pueden hacer mucho para aumentar la autoestima de sus alumnos, si bien la participación de los padres en este aspecto es crucial, ya que, por encima de todo, los niños se desviven por impresionar a sus padres y ser queridos, aceptados por ellos. Ahora bien, si el niño que asiste a la escuela posee poca autoestima, es probable que los padres (ya sean biológicos, adoptivos o meros agentes de crianza) tengan asimismo dificultades con su propia autoestima. Los padres y los profesores que tengan una autoestima alta transmitirán este mismo grado de autoestima a los niños, aunque no se debe olvidar que también puede suceder el caso contrario. Este proceso sucede independientemente de si los padres y demás adultos importantes en la vida del niño o de la niña se den o no cuenta de él. Toda acción o expresión facial, así como cualquier interacción gestual o verbal que tengan con sus mayores, transmitirá a los niños algún tipo de mensaje sobre su talento, valía y capacidad.

Es importante porque tiene que ver con el rendimiento escolar, con la motivación, con el desarrollo de la personalidad, con las relaciones sociales y con el contacto afectivo del niño consigo mismo.

Es necesario que los profesores y los padres, por su enorme significación para los niños, asuman un rol activo en el desarrollo de una autoestima positiva y tomen conciencia de los efectos emocionales que tienen la aprobación y el rechazo. 
Cada vez que se establece una relación, se está transmitiendo aprobación o desaprobación y, en esa misma medida, se van devolviendo o entregando características personales que pasan a integrar la autoimagen de esa persona.

De este modo, la interacción con el profesor va teniendo repercusiones en el sentimiento de confianza en sí mismo que desarrolla el niño, es decir, si siente que lo hace bien o mal.

Esta interacción es también un modelo acerca de cómo se establecen relaciones interpersonales. Los niños van internalizando modelos de interacción que implican una tendencia a confirmar o descalificar al otro.

Si el niño percibe que el profesor es cercano, acogedor y valorativo con los alumnos, va a introyectar formas de establecer relaciones interpersonales con estas características. Si por el contrario observa y aprende formas distantes, críticas o descalificatorias de relacionarse, interiorizará en forma casi automática este tipo de interacciones.

Se puede apreciar que existe una relación circular, si el niño tiene una autoestima alta, se comportará en forma agradable, será cooperador, responsable, rendirá mejor y facilitará el trabajo escolar. Por lo tanto, el profesor posiblemente será reforzante, estimulante y entregará retroalimentación positiva, lo que hará que el niño se comporte mejor; y así sucesivamente, generándose un círculo virtuoso.

Si su autoestima es baja se pondrá agresivo, irritable, poco cooperador, poco responsable. En esta situación es altamente probable que el profesor tienda a asumir una postura más crítica y rechazante frente al niño, quien, a su vez, se pondrá más negativo y desafiante, creándose así un circulo vicioso.

Por otro lado se ha encontrado una relación entre la autoestima de los profesores y la autoestima de los niños. Reasoner (1982), por ejemplo, señala que los profesores con una buena autoestima dan más seguridad a los niños, están más satisfechos con su rendimiento escolar, desarrollan un clima emocional más positivo y sus alumnos se aprecian más contentos en la sala de clases. Los profesores con baja autoestima tienden a tener miedo de perder autoridad, por lo tanto usan una disciplina mucho más represiva; sus alumnos son menos creativos, no saben trabajar solos, son más tensos e irritables y dependen del control que el adulto ejerce sobre ellos.

\section{La autoestima y las dificultades de aprendizaje en los niños}

La forma en que los padres respondan a los problemas de autoestima de sus hijos estará principalmente determinada por sus propios niveles de autoestima. Cuando son los mismos padres quienes tienen dudas acerca de su propio valor y capacidad existe la tendencia por parte de éstos a ser sobreprotectores o exigentes en demasía; aunque también se puede dar el caso de que lleguen incluso a desentenderse de sus hijos. Esto da como resultado que los hijos tengan también dificultades de autoestima.

Todos los niños desean complacer a sus padres, por lo que la posibilidad de una humillación debida a la crítica y a la negación del cariño producirá en ellos dos posibles reacciones. Una reacción es la apatía y la inhibición. En este caso, el niño se abstiene de realizar cualquier esfuerzo académico o de otro tipo porque sabe que el intentarlo conlleva el riesgo de una humillación o de un rechazo. El niño subconscientemente razona así: «Si me abstengo de hacerlo, no fallo y si no fallo no hay humillación». 
La otra reacción de los niños cuando ven amenazada su autoestima es la compensación. Esto se hace evidente en el niño o la niña que es trabajador, perfeccionista, que dedica muchas horas.

\section{Relaciones entre la autoestima y rendimiento escolar}

Diferentes estudios han podido demostrar la existencia de una estrecha relación entre autoestima y rendimiento escolar.

Como es evidente, por lo general los niños con buen rendimiento escolar tienen una buena autoestima. Ellos tienden a confiar en sus capacidades y a sentirse autoeficaces y valiosos.

Se ha observado que la autoestima académica, es decir, el niño se percibe a sí mismo en la situación escolar, tiene un peso muy importante dentro de la autoestima global. Así, durante la etapa escolar, los niños que tienen un buen desempeño académico, por lo general no presentan problemas en su autoestima, puesto que, dados sus logros escolares y la importancia de estos, tienden a sentirse bien con ellos mismos.

Los niños de bajo rendimiento escolar, en cambio, tienden a presentar una baja motivación por aprender, a esforzarse poco, a quedarse con una sensación de frustración por sus experiencias de fracaso, a sentirse poco eficaces y a evitar los desafíos escolares, puesto que parten pensando que no les va ir bien. Estos alumnos presentan generalmente una autoestima baja. Cuando logran tener algún éxito, lo atribuyen a factores externos: «tuve suerte» o «era fácil la prueba», y sus fracasos, a su falta de habilidad: «no puedo», «no soy capaz». Según los estudios realizados, este tipo de atribución, además de tener un alto costo emocional y ser un fracaso para el desarrollo de la autoestima, dificulta la superación académica, ya que el alumno asume una actitud desesperanzada por falta de motivación y energía para el trabajo.

En relación a la familia de los niños con trastornos específicos, los estudios de Bryan, Peral, Zimmerman y Mattew (1982) refieren que en una familia etiquetadora o abandonadora, los niños pierden el interés, bajan su rendimiento y están constantemente confirmando con su actitud negativa la baja opinión que se tienen de ellos. No obstante también han mostrado que si la familia apoya al niño mostrándole confianza en sus capacidades y dándole el apoyo necesario, pese a las dificultades, el niño logra mantener una autoestima adecuada.

De este modo, el colegio y la familia pueden ser instituciones que respondan apropiadamente a las necesidades del niño, logrando evitar que se produzcan efectos emocionales secundarios a las dificultades de rendimiento escolar. Jain y Zimmerman (1982, citado en Fish y Jain, 1983) aislaron cuatro variables significativas en este sentido, al comparar familias de niños que presentaban problemas derivados de los trastornos de aprendizaje, pero sin problemas emocionales. Las cuatro variables descritas por estos autores son las siguientes:

a. Cohesión del grupo familiar.

b. Aceptación del trastorno de aprendizaje.

c. Apoyo emocional.

d. Compensación activa de las áreas deficitarias.

Con relación a la cohesión del grupo familiar, se señala que los niños con trastornos de aprendizaje, pero sin problemas emocionales provienen mayoritariamente de familias donde 
hay una estrecha unión de la pareja de padres y donde existen límites entre el subsistema padres y el subsistema hijos, es decir, donde los padres no se dejan invadir por los hijos.

Lo anterior enfatiza la necesidad de establecer límites entre ambos subsistemas. Además, se aprecia que en las familias de niños sin problemas emocionales, la figura paterna se involucra más activamente en el aprendizaje de sus hijos. Estos destacan la importancia de comprometer más al padre en el rendimiento escolar de los niños.

En cuanto a la aceptación del trastorno del aprendizaje, los padres de los niños sin dificultades emocionales conocen y aceptan abiertamente el problema de sus hijos y lo localizan en un área, no generalizando la dificultad a todo el comportamiento del niño.

En los niños bien adaptados, el apoyo emocional se materializa en esfuerzos sostenidos y sistemáticos de los padres para disminuir el efecto del fracaso y lograr el desarrollo de otras habilidades, de manera que su imagen personal no dependa exclusivamente del colegio.

En relación a la compensación de las dificultades, los padres de los niños sin problemas emocionales tienden a ser más activos en diseñar estrategias para ayudarlos a superar su problemática, tienen expectativas claras y toman en cuenta los obstáculos que el niño enfrenta, no atribuyéndolos exclusivamente a la falta de esfuerzo.

En el contexto escolar, algunas dificultades emocionales que suelen presentar los niños con problemas de aprendizaje son:

a. Aceptación del trastorno del aprendizaje por parte de los profesores.

b. Apoyo emocional entregado a los estudiantes.

c. Compensación activa de las áreas deficitarias a través de diferentes estrategias pedagógicas, por ejemplo diseñar, situaciones en las que estos alumnos puedan ser altamente exitosos, o desarrollar programas de tutorías que le permitan asimilar la información en forma más individual.

Es en base a estas consideraciones se plantea la siguiente interrogante: ¿Cuál es el efecto que produce el programa para el mejoramiento de la autoestima en niños de 8 a11 años con problemas específicos de aprendizaje?

\section{Objetivos}

a. Diseñar un programa para el mejoramiento de la autoestima en los niños con problemas específicos de aprendizaje.

b. Aplicar un programa para el mejoramiento de la autoestima en los niños con problemas específicos de aprendizaje.

c. Evaluar el efecto de un programa para el mejoramiento de la autoestima en niños con problemas específicos de aprendizaje.

\section{Hipótesis}

$\mathrm{H}_{1}$. Los niños que participan en el programa presentan un mayor desarrollo en el área de sí mismo general que los niños del grupo de control. 
$\mathrm{H}_{2}$. Los niños que participan en el programa presentan un mayor desarrollo en el área de hogar que los niños del grupo de control.

$\mathrm{H}_{3}$. Los niños que participan en el programa presentan un mayor desarrollo en el área de escuela que los niños del grupo de control.

$\mathrm{H}_{4}$. Los niños que participan en el programa presentan un mayor desarrollo en el área de pares que los niños del grupo de control.

$\mathrm{H}_{5}$. Los niños que participan en el programa presentan un mayor desarrollo en la Autoestima total que los niños del grupo de control.

\section{MÉTODO}

La presente investigación corresponde a un estudio Experimental (Alarcón, 1991, Sánchez y Reyes, 2000), debido a que se trata de estudiar el efecto que produce un programa de mejoramiento de la autoestima en niños con problemas específicos de aprendizaje.

\section{Diseño de investigación}

Se aplicó un diseño de investigación experimental con grupo de control con pretest y posttest. (Alarcón, 1991; Hernández, Fernández y Baptista, 1998; Sánchez y Reyes, 2002). Su diagrama es el siguiente:

$$
\begin{array}{lll}
\text { GE } & \mathrm{O}_{\mathrm{x} 1} & \mathrm{O}_{\mathrm{x} 2} \\
\mathrm{GC} & \mathrm{O}_{\mathrm{x} 3} & \mathrm{O}_{\mathrm{x} 4}
\end{array}
$$

En el esquema:

$\mathrm{GE}=$ Grupo experimental

$\mathrm{GC}=$ Grupo de control.

$\mathrm{O}_{\mathrm{x} 1}, \mathrm{O}_{\mathrm{x} 2}, \mathrm{O}_{\mathrm{x} 3}, \mathrm{O}_{\mathrm{x} 4}=$ Variables observadas.

\section{Variables}

Variable Independiente Manipulada:

Programa de mejoría de la autoestima.

\section{Variable dependiente}

Autoestima y las áreas de sí mismo, hogar, escuela, pares y la autoestima total, evaluadas a través de la escala de autoestima de Coopersmith para niños.

\section{Variables controladas}

Presencia de problemas específicos de aprendizaje.

Sexo.

Edad : niños de 8 a 11 años. 


\section{Participantes}

La población estuvo conformada por los niños que asisten al Departamento de Discapacidades de aprendizaje del Instituto Nacional de Rehabilitación, que tuvieron el diagnóstico de Problema específico de aprendizaje, con edades entre 8 y 11 años y que cursaban el tercer y el quinto grado de primaria.

La muestra estuvo determinada de acuerdo a consideraciones estadísticas y puesto que se utilizó para el contraste de las hipótesis la prueba t de student, el tamaño mínimo de cada grupo para obtener diferencias significativas es de 15 casos (Anastasi, 1998) Es por ello que el grupo experimental y el grupo de control estuvo conformado por 15 sujetos.

\section{Instrumentos}

Escala de autoestima de Coopersmith para escolares

Evalúa los siguientes aspectos:

- Sí Mismo

- Hogar

- Escuela

- Pares

- La Autoestima total.

La prueba en su versión escolar fue adaptada por Ursula Busse en 1997.

\section{Procedimiento experimental}

El objetivo general del programa de mejoramiento de la autoestima es: Promover, desarrollar e incrementar los niveles de autoestima en niños que presentan problemas específicos de aprendizaje.

Los objetivos específicos son:

1. Fomentar la integración, comunicación y socialización entre los participantes y coordinadores, así como iniciar el conocimiento de la autoestima.

2. Desarrollar la capacidad de autoconocimiento de los aspectos positivos y negativos de su personalidad así como de las otras personas.

3. Conocer y mejorar las ideas irracionales acerca de sí mismo y de los demás.

4. Fomentar la capacidad de aceptar su forma de ser, sus habilidades y destrezas que le permiten obtener logros.

5. Fomentar el manejo y el reconocimiento de sus necesidades y valores. Así como la expresión de sus sentimientos y emociones que lo hagan sentirse orgulloso de sí mismo.

6. Mejorar el nivel de autoestima en el área hogar de los niños que presentan problemas específicos de aprendizaje. 
7. Mejorar el nivel de autoestima en el área escuela de los niños con problemas específicos de aprendizaje.

8. Reforzar las actividades realizadas y brindar al niño un mensaje que lo anime a poner en práctica lo aprendido acerca de la autoestima.

La meta que se propuso al finalizar el programa es que el $95 \%$ de los participantes logren incrementar su nivel de autoestima.

El programa estuvo conformado por 9 sesiones que fueron aplicadas una vez por semana. Cada sesión estuvo dividida en tres partes o momentos: motivación, desarrollo de la actividad básica, despedida y evaluación de la sesión. El tiempo de duración de cada sesión fue de aproximadamente dos horas.

La metodología del programa fue eminentemente participativa, ya que se buscó lograr el intercambio de alaternativas y experiencias entre los participantes así como el aprendizaje en forma grupal.

Procedimiento de recogida de datos

Se realizó en tres momentos.

a. Pretest. Se evaluaron los dos grupos antes del desarrollo del programa. Las evaluaciones debido a las edades de los participantes se pudieron realizar de forma grupal.

b. Aplicación del programa al grupo experimental.

c. Postest, se evaluó a los dos grupos después de finalizado el programa.

\section{RESULTADOS}

El análisis estadístico fue realizado tomando en cuenta las recomendaciones propuestas por Siegel (1995), el cual abarcó las siguientes etapas:

En primer lugar se exponen los resultados obtenidos del análisis estadístico de la distribución de las puntuaciones de la escala a través de la prueba de bondad de ajuste a la curva normal de Kokmogorov-Smirnov.

En segundo lugar se presentan los hallazgos del contraste de las hipótesis de cada una de las áreas desarrolladas en el programa de intervención a través de la prueba t de student

Finalmente, se presenta el análisis comparativo de la escala de autoestima de Coopersmith entre el pre y el postest del grupo experimental.

El análisis de la distribución de las puntuaciones de la escala de autoestima de Coopersmith, efectuado a través de la prueba de Kolmogorov-Smirnov (Tabla 1), indica que en todos los casos no existen diferencias significativas en los estilos evaluados, lo que nos permite concluir que los puntajes tienen una adecuada distribución que se aproxima a la curva normal y por lo tanto efectuar los análisis inferenciales utilizando estadísticas paramétricas. 
Tabla 1. Test de Bondad de Ajuste a la Curva Normal de Kolmogorov-Smirnov de la Escala de Autostima de Coopersmith.

\begin{tabular}{|l|c|c|c|}
\hline \multicolumn{1}{|c|}{ Estilo } & Media & D. S. & K-S Z \\
\hline Sí mismo & 7.73 & 2.41 & 0.89 \\
\hline \multicolumn{4}{|c|}{} \\
\hline Hogar & 3.77 & 1.38 & 1.36 \\
\hline \multicolumn{4}{|c|}{} \\
\hline Escuela & 1.83 & 1.02 & 0.56 \\
\hline Pares & 2.57 & 1.22 & 1.35 \\
\hline \multicolumn{4}{|c|}{} \\
\hline Total Autoestima & 15.90 & 4.56 & 0.99 \\
\hline
\end{tabular}

$$
\begin{aligned}
& p<0.05 \\
& n=30
\end{aligned}
$$

El análisis comparativo del pretest entre el grupo experimental y el grupo de control, presentado en la Tabla 2, indica que no existen diferencias estadísticamente significativas entre ninguna de las áreas ni el puntaje total de la autoestima, lo que permite confirmar que los grupos son similares al inicio del estudio.

Tabla 2. Análisis comparativo de la escala de autoestima de Coopersmith por grupo de estudio en

\begin{tabular}{|c|c|c|c|c|c|}
\hline \multirow[t]{2}{*}{ Escala } & \multicolumn{2}{|c|}{$\begin{array}{c}\text { Grupo } \\
\text { Experimental } \\
n=15\end{array}$} & \multicolumn{2}{|c|}{$\begin{array}{l}\text { Grupo de Control } \\
\qquad n=15\end{array}$} & \multirow[t]{2}{*}{$\mathbf{t}$} \\
\hline & $\mathbf{M}$ & D. S. & M. & D. S. & \\
\hline Sí mismo & 7.67 & 2.44 & 7.80 & 2.46 & -0.15 \\
\hline Hogar & 4.13 & 1.19 & 3.40 & 1.50 & 1.48 \\
\hline Escuela & 1.87 & 0.99 & 1.80 & 1.08 & 0.18 \\
\hline Pares & 2.67 & 1.29 & 2.47 & 1.19 & 0.44 \\
\hline Total Autoestima & 16.33 & 4.39 & 15.47 & 4.84 & 0.51 \\
\hline
\end{tabular}
los pretest a través de la prueba t de Student.

$$
\begin{aligned}
& p<0.05 \\
& n=30
\end{aligned}
$$

El análisis comparativo del postest entre el grupo experimental y el grupo de control (Tabla 3) denota la existencia de diferencias estadísticamente significativas en: Sí mismo $(t=2.10$, $\mathrm{p}<.05)$, Hogar $(\mathrm{t}=3.49, \mathrm{p}<.01)$, Escuela $(\mathrm{t}=3.69, \mathrm{p}<.01)$, Pares $(\mathrm{t}=3.31, \mathrm{p}<$ .01 ) y en el Total de Autoestima $(\mathrm{t}=4.46, \mathrm{p}<.001)$ pudiendo observarse que en todos los casos los resultados alcanzados en el postest por el grupo experimental es mayor que el 
alcanzado por el grupo de control, lo que permite validar las hipótesis H1, H2, H3, H4 y $\mathrm{H} 5$, por lo que se comprueba la eficiencia del programa para mejorar la autoestima.

Tabla 3. Análisis comparativo de la escala de autoestima de Coopersmith por grupo de estudio en los postest a través de la prueba t de Student.

\begin{tabular}{|c|c|c|c|c|c|}
\hline \multirow[t]{2}{*}{ Escala } & \multicolumn{2}{|c|}{$\begin{array}{l}\text { Grupo Experimental } \\
\qquad N=15\end{array}$} & \multicolumn{2}{|c|}{$\begin{array}{c}\text { Grupo de Control } \\
\qquad N=15\end{array}$} & \multirow[t]{2}{*}{$\mathbf{t}$} \\
\hline & M & D. S. & M. & D. S. & \\
\hline Sí mismo & 9.33 & 1.40 & 7.80 & 2.46 & $2.10 *$ \\
\hline Hogar & 4.93 & 0.80 & 3.40 & 1.50 & $3.49 * *$ \\
\hline Escuela & 3.60 & 1.55 & 1.80 & 1.08 & $3.69 * *$ \\
\hline Pares & 3.87 & 1.13 & 2.47 & 1.19 & $3.31 * *$ \\
\hline Total Autoestima & 21.73 & 2.49 & 15.47 & 4.84 & $4.46 * * *$ \\
\hline
\end{tabular}

${ }^{*} p<0.05{ }^{* *} p<0.01{ }^{* * *} p<.001$

El análisis comparativo entre el pretest y el postest, presentado en la Tabla 4, permite apreciar que existen diferencias estadísticamente significativas en las escalas de: Si mismo $(\mathrm{t}=-4.06, \mathrm{p}<.01)$, Hogar $(\mathrm{t}=-2.26, \mathrm{p}<.05)$, Escuela $(\mathrm{t}=-3.45, \mathrm{p}<.01)$, Pares $(\mathrm{t}$ $=-3.06, \mathrm{p}<.01)$ y en el Total de Autoestima $(\mathrm{t}=-6.23, \mathrm{p}<.001)$, notándose en todos los casos que el post-trest es mayor que el pre-test, lo que corrobora la efectividad del programa de mejoramiento de la autoestima.

Tabla 4. Análisis comparativo de la escala de autoestima de Coopersmith entre el pretest y el postest del grupo experimental.

\begin{tabular}{|c|c|c|c|c|c|}
\hline \multirow{2}{*}{ Escala } & \multicolumn{2}{|c|}{ Pretest } & \multicolumn{2}{|c|}{ Postest } & \multirow{2}{*}{$\mathbf{t}$} \\
\hline & $\mathbf{M}$ & D. S. & M. & D. $\mathbf{S}$. & \\
\hline Sí mismo & 7.67 & 2.44 & 9.33 & 1.40 & $-4.06 * *$ \\
\hline Hogar & 4.13 & 1.19 & 4.93 & 0.80 & $-2.26 *$ \\
\hline Escuela & 1.87 & 0.99 & 3.60 & 1.55 & $-3.45 * *$ \\
\hline Pares & 2.67 & 1.29 & 3.87 & 1.13 & $-3.06 * *$ \\
\hline Total Autoestima & 16.33 & 4.39 & 21.73 & 2.49 & $-6.23 * * *$ \\
\hline
\end{tabular}

${ }^{*} p<0.05 \quad{ }^{* *} p<0.01 \quad{ }^{* * *} p<.001$

$n=15$ 


\section{DISCUSIÓN}

Sin duda que los resultados presentados abren una serie de interrogantes, los cuales pueden ser ordenados en función de las hipótesis tal como se hace a continuación.

La primera hipótesis específica señala que los niños que participan en el programa presentan un mayor desarrollo en el área de sí mismo que los niños del grupo de control por lo que se acepta esta hipótesis. El reconocer y apreciar la propia valía significa ser consciente no solo de la propia forma de actuar en un momento dado, sino también de los propios potenciales y posibilidades. El ser responsable hacia sí mismo significa aceptar que cada uno asume concientemente las propias acciones y por lo tanto también las consecuencias de las mismas.

Con respecto a la segunda hipótesis específica que señala que los niños que participan en el programa presentan un mayor desarrollo en el área hogar que los niños del grupo control por lo que se acepta la hipótesis. La familia satisface predominantemente las necesidades emocionales de los niños. El núcleo familiar le sirve al niño de punto de referencia donde establece sus relaciones más intimas.

La tercera hipótesis específica señala que los niños que participan en el programa presentan un mayor desarrollo en el área escuela que los grupos de control, por lo que se acepta esta hipótesis. Sin duda la experiencia escolar será un factor determinante para el desarrollo de la autoestima dado el gran impacto de las experiencias de rendimiento escolar. Según Milicic (1995) la experiencia escolar determinará en forma importante el bienestar socioemocional de un niño y tendrá efectos significativos durante la vida adulta.

Referente a la cuarta hipótesis que señala que los niños que participan en el programa presentan un mayor desarrollo en el área de pares que los niños del grupo de control es posible señalar que se acepta esta hipótesis. Otro factor que incidirá en el desarrollo de sí mismo es la interacción del niño con el grupo de pares. Esta se ve influenciada entre otros factores por el éxito o fracaso que existe dentro del grupo. Así los alumnos más capaces tendrán un sitial especial respecto de la clase y los que han tenido reiterados fracasos serán relegados a un segundo plano.

Finalmente la quinta hipótesis plantea que los niños que participan en el programa presentan un mayor desarrollo en la autoestima total que los niños del grupo control por lo que se acepta la hipótesis. Según Branden (1994), una autoestima saludable se relaciona con la racionalidad, el realismo con la creatividad, la independencia, la flexibilidad y la capacidad para aceptar los cambios con el deseo de admitir los errores y la disposición a cooperar. Mientras que la autoestima baja se correlaciona con la irracionalidad y la ceguera ante la realidad, con la rigidez, el miedo a lo nuevo. Todas estas características tienen implicancias para la realización personal y la adaptación social.

\section{CONCLUSIONES}

Los resultados de la investigación indican que:

1. La hipótesis $\left(\mathrm{H}_{1}\right)$ es valida, puesto que los niños que participaron en el programa mejoraron en el área de sí mismo.

2. Se acepta la hipótesis $\left(\mathrm{H}_{2}\right)$, puesto que los niños que participaron en el programa mejoraron en el área hogar. 
3. La hipótesis $\left(\mathrm{H}_{3}\right)$ es válida en la medida de que los niños que participaron en el programa mejoraron en el área escuela.

4. Se acepta la hipótesis $\left(\mathrm{H}_{4}\right)$ en la medida de que los niños que participaron en el programa presentan un mayor desarrollo en el área pares.

5. Se acepta la hipótesis $\left(\mathrm{H}_{5}\right)$ en la medida de que los niños que participaron en el programa presentan un mejor desarrollo en la autoestima total, lo que corrobora la efectividad del programa.

\section{REFERENCIAS BIBLIOGRÁFICAS}

1. Alarcón, R. (1991). Métodos y diseños de investigación del comportamiento. Lima: Universidad Peruana Cayetano Heredia, Fondo Editorial.

2. Anastasi, A., y Urbina, S. (1998). Test psicológicos. México: Ed. Prentice Hall.

3. Branden, N. (1994). Los seis pilares d e la autoestima. Madrid: Ed. Paidós.

4. Céspedes, N. (1977). Escuela de padres: La autoestima en los niños y niñas. Lima: Ed. Tarea.

5. Céspedes, N. (1998). Para ser mejores: La autoestima en la escuela. Lima: Ed. Tarea.

6. Fredes, A. (1998). Autoestima y sus efectos en el ámbito escolar. Santiago de Chile: Ed. CPEIP.

7. Hernández, R.; Fernández,C.; Baptista,P. (1998). Metodología de la investigación. México: Ed Mac. Graw-Hill.

8. Lindenfield, G. (1998). Como desarrollar la autoestima en niños y adolescentes. Madrid: Ed. Neo Person.

9. Milicic, N. (1995). La autoestima en los niños: un programa de desarrollo emocional. Santiago de Chile: Ed.Domen.

10. Sánchez, C. H. y Reyes, M. C. (2002). Metodología y Diseños en la Investigación Científica: Aplicadas a la Psicología, Educación y Ciencias Sociales. Lima: Ed. URP.

11. Siegel, S. y Castellan N. (1995). Estadística no paramétrica: Aplicada a las ciencias de la conducta. México: Ed. Trillas.

12. Reasoner, R. (1982). Building sel-esteem: teacher's guide and cassroom materials california. Consulting Psychologist Press, Inc.

13. Rodríguez, M. (1996). Autoestima clave para el éxito personal. México: Ed. Trillas.

14. Vollo, F. (1998). La autoestima del adolescente. Buenos Aires: Ed. Paidós. 